\title{
Brain Injury is a Major Problem in Canada and Annual Incidence is Not Declining
}

Brain injuries, both catastrophic and non-catastrophic, are a major public health concern, worldwide. In North America, brain injuries are a major component of the overall trauma burden, perhaps the single most costly item. Indeed, catastrophic brain injuries account for about half the trauma deaths ${ }^{1}$ and about half the trauma cases with permanent, major disability. Recently, concussions have also received appropriate attention because they are now correctly recognized as brain injuries, are very frequent, and although generally non-catastrophic, they too can lead to catastrophic disablement from depression and dementia ${ }^{2,3}$. The Canadian Brain and Nerve Health Coalition was initiated by the Canadian Neurological Sciences Federation and partnered with the Canadian Institute for Health Information (CIHI) in a study that showed traumatic brain injuries (TBI) are a significant portion of the burden of neurological conditions in Canada ${ }^{4}$.

Colantonio and colleagues, in this issue of the Journal, confirmed the continuing high annual incidence of TBI in Ontario, Canada ${ }^{5}$. Their report also shows that even in Canada with its government controlled, single payer system there are still major difficulties in collecting annual incidence data and calculating rates of brain injuries. We have inherited some flawed systems for defining and counting cases of trauma and TBI that make it difficult to determine the exact annual incidence of TBI, but our system is still ahead of many countries because of national health care, and the trauma registries that our governments have supported. For example, we have an improved system for counting hospitalized trauma cases, with the important antecedent being the Ontario Trauma Registry now broadened into the National Trauma Registry, both of which are now under the umbrella of CIHI. Also, the National Ambulatory Care Resource System was recently added for emergency department (ED) cases (although not yet Canada-wide). The combination of hospitalization and ED data is essential for determining the true annual incidence of TBI, as shown by Colantonio et $\mathrm{al}^{5}$. However, even this combination still omits cases that only go to doctors' offices outside hospitals and EDs. Indeed, based on my own practice, it is highly likely that a very large number of TBI cases, especially concussions (perhaps the majority of concussions) are not hospitalized and never go to an $\mathrm{ED}$, and therefore, are not captured in the data presented by Colantonio and colleagues ${ }^{5}$. The "injury pyramid" for all types of trauma is well recognized with death at the top, then hospitalization, followed by the vast number of cases seen in EDs, then doctors' offices, and at the bottom those without any medical attention ${ }^{6}$. Another category excluded by Colantonio et al were those who died before reaching hospital which represent an important fraction ${ }^{7}$.
Even with the above limitations most of which were acknowledged by the authors, their combined data on hospitalizations and ED visits provide much useful information ${ }^{5}$. For example, they showed clearly that three times as many TBI patients visit EDs than get admitted to hospitals, and that the total number of TBIs did not decrease during the 2002-2007 period of their study. Thus, the annual incidence of TBI has not decreased, whereas a decrease had been suggested by the 2007 CIHI report showing declining TBI hospitalization rates ${ }^{4}$. At that time, many attributed the decline in TBI hospitalization to major practice changes mainly the greater availability of computerized tomographic scans, rather than to a true decline in the annual incidence of TBI. A normal computerized tomographic scan permitted clinicians to discharge milder TBI patients from the ED avoiding hospital admission for observation, formerly frequently necessary for safe management. Indeed, the 2007 CIHI report generated a ThinkFirst Canada symposium on TBI incidence at which declining hospitalization for TBI was thought primarily due to significant practice changes ${ }^{8}$. The report by Colantonio and colleagues confirms that the annual incidence of TBI is not declining 5 .

The Colantonio et al report is also noteworthy because it describes the mechanisms and causes of injury more accurately than other TBI reports ${ }^{5}$. Typical reports of this type use broad categories such as "motor vehicle accidents" that include diverse activities such as cars and trucks on highways and snowmobiles and ATVs on trails, and "falls" that include diverse events such as a construction worker falling off a 40-storey building, a 95year-old falling downstairs at home, and a child falling off a trampoline. Such broad categories are of little value for injury prevention purposes. In contrast, the present authors categorized injuries much more precisely. For example, cycling injuries and sports injuries were each separate categories. Indeed, sports injuries accounted for $20.2 \%$ of brain injuries, and intentional injuries accounted for $11.0 \%$. These data are of utmost importance for injury prevention programs that must be targeted toward specific populations to achieve maximum effectiveness. These are costly categories for our country. For example, sports injuries in Ontario, with head injury as the most frequent catastrophic injury, cost Ontarians about $\$ 3$ billion annually, and most are preventable ${ }^{9}$.

The Colantonio et al report also confirms some important demographic data about TBI patients, especially the overall preponderance of males in terms of number of cases per year, especially young males in the up to 24 -year-old age group 5 . Indeed, it was astounding that more than half the cases of TBI in males and slightly less than half the cases in females were 24 or less. This information is very useful for injury prevention 
organizations to continue targeting this very difficult and injury prone age group. With respect to injury rates per 100,000 population, older age groups had much higher rates due to the shrinking denominators with ageing. These important differences in injury rates are also essential for injury prevention programs. Although the authors alluded to the role of governments in injury prevention in Canada, the vast majority of Canadian injury prevention programs are conducted by nongovernmental organizations like ThinkFirst, Safe Kids, Smart Risk, Safe Communities and others. Indeed, it is fitting that the report by Colantonio et al appears in the Canadian Journal of Neurological Sciences because Canadian neurosurgeons and neurologists play a major role in injury prevention.

Charles H. Tator Toronto Western Hospital and University of Toronto Toronto, Ontario, Canada Founder, ThinkFirst Canada

\section{REFERENCES}

1. Kraus J. Epidemiology of head injury. In: Cooper P, editor. Head injury. 3rd ed. Baltimore: Williams and Wilkins; 1993. p. 1-25.

2. McKee AC, Cantu RC, Nowinski CJ, et al. Chronic traumatic encephalopathy in athletes: progressive tauopathy after repetitive head injury. J Neuropathol Exp Neurol. 2009;68:709-35.

3. De Beaumont L, Theoret $\mathrm{H}$, Mongeon $\mathrm{D}$, et al. Brain function decline in healthy retired athletes who sustained their last sports concussion in early adulthood. Brain. 2009;132:695-708.

4. The burden of neurological diseases, disorders and injuries in Canada: Head injury. Ottawa: The Canadian Institute of Heath Information (CIHI); 2007.

5. Colantonio A, Saverino C, Zagorski B, et al. Hospitalizations and emergency department visits for TBI in Ontario. Can J Neurol Sci. 2010;37(6):783-90.

6. Bishai D, Gielen A. How much outpatient care is provided for injuries? Inj Prev. 2001;7:70-3.

7. Tator CH. Overview of the results of the Ontario study, 1986-1995. In: Tator $\mathrm{CH}$, editor. Catastrophic injuries in sports and recreation, causes and prevention: a Canadian Study. Toronto: University of Toronto Press; 2008. p. 21-57.

8. Tator $\mathrm{CH}$. Measuring the incidence and prevalence of head injuries in Canada. Paper presented at: ThinkFirst Summit on Head Protection. Toronto; 2007.

9. Provvidenza C, Tator CH. Sports injuries prevention: general principles. In: Tator $\mathrm{CH}$, editor. Catastrophic injuries in sports and recreation, causes and prevention: a Canadian study. Toronto: University of Toronto Press; 2008. p. 58-78. 